\title{
Derivation of Closed-Form Green's Functions for a General Microstrip Geometry
}

\author{
M. Irsadi Aksun and Raj Mittra, Fellow, IEEE
}

\begin{abstract}
The derivation of the closed-form spatial domain Green's functions for the vector and scalar potentials is presented for a microstrip geometry with a substrate and a superstrate, whose thicknesses can be arbitrary. The spatial domain Green's functions for printed circuits are typically expressed as Sommerfeld integrals, that are inverse Hankel transform of the corresponding spectral domain Green's functions, and are quite time-consuming to evaluate. Closed-form representations of these Green's functions in the spatial domains can only be obtained if the integrands are approximated by a linear combination of functions that are analytically integrable. In this paper, we show we can accomplish this by approximating the spectral domain Green's functions in terms of complex exponentials by using the least square Prony's method.
\end{abstract}

\section{INTRODUCTION}

$\mathrm{T}$ HE RIGOROUS analysis of printed circuit elements, such as microstrip interconnects terminated by complex loads, microstrip discontinuities, patch antennas and printed dipoles, requires the use of the vector and scalar Green's functions for a substrate layer backed by a ground plane. It is well-known that the Green's functions for microstrip geometries are improper integrals [1], also called Sommerfeld integrals, whose integrands are oscillatory and slowly decaying functions; hence, their calculation is very time-consuming if not impractical for many practical configurations of interest. However, recently a novel approach to circumventing this problem has been developed [2], [3], one that employs closed-form expressions for the spatial domain Green's functions corresponding to the vector and scalar potentials associated with a horizontal electric dipole (HED) located over a thick substrate. In this paper, this technique is extended to a general class of microstrip geometries with both a substrate and a superstrate, and the restriction imposed on the thickness of the substrate is relaxed by slightly modifying the original procedure.

Using the closed-form expressions for the spatial domain Green's functions in a variational technique, e.g., the method of moments (MoM), can result in a substantial savings of computation time when analyzing planar microstrip structures. Once the improper infinite range in-

Manuscript received November 14, 1991 ; revised March 30, 1992.

M. I. Aksun was with the Electromagnetic Communication Laboratory, Department of Electrical and Computer Engineering, University of Illinois at Urbana-Champaign, Urbana, IL 61801 . He is presently with the Department of Electrical and Electronics Engineering, Bilkent University, 06533, Ankara, Turkey.

R. Mittra is with the Electromagnetic Communications Laboratory, Department of Electrical Engineering, University of Illinois at Urbana-Champaign, Urbana, IL 61801 .

IEEE Log Number 9202899 tegrals for Green's functions have been expressed in closed-forms, the rest of the integrals need only be computed over finite supports associated with the basis and testing functions. In view of this, it would be instructive to demonstrate the difficulties that one may face in the application of the conventional moment method approach to microstrip geometries, before starting the derivation of the closed-form Green's functions for the vector and scalar potentials. It is well-known that MoM can be applied either in the spatial domain [4] or in the spectral domain [5], the latter being more suitable for planar geometries unless the spatial domain Green's functions can be approximated in closed-forms [6], [7]. Both approaches will be briefly examined here with a view to comparing their computational efficiency.

An important issue that merits examination in the MoM formulation is the convergence problem of the integrals representing the MoM matrix. The expressions of these elements in the spatial and spectral domains are given in Section II and the difficulties associated with their evaluation are discussed. This is followed in Section III with the derivation of the closed-form Green's functions of the vector and scalar potentials for a microstrip geometry with a substrate and superstrate, whose thickness are arbitrary A discussion of the technique employed for the derivation of the closed-form expressions and some numerical examples are included in Section IV.

\section{Computational Difficulties in the Conventional Method of Moments}

Consider, for the sake of illustration, a general microstrip structure shown in Fig. 1 where it is assumed that the substrate layer extends to infinity in the transverse directions. Let the thickness and the permittivity of the substrate be denoted by $d$ and $\epsilon_{r}$, respectively. Although the Green's functions discussed herein pertain to the geometry shown in Fig. 1, the comments appearing in this section apply to more general geometries as well. A time convention of $e^{j \omega t}$ has been adopted in this work.

\section{A. Spatial Domain Analysis}

The tangential electric fields on the plane of the patch $(z=0)$ can be written in terms of the surface current density $J$ and the Green's functions for the vector and scalar potentials $\tilde{G}_{x x}^{A}$ and $\tilde{G}_{q}$, respectively;

$$
E_{x}=-j \omega G_{x x}^{A} * J_{x}+\frac{1}{j \omega} \frac{\partial}{\partial x}\left[G_{q} * \nabla \cdot J\right]
$$




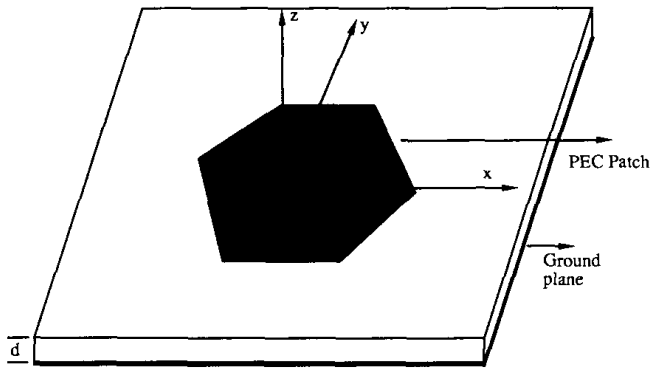

Fig. 1. A general microstrip structure.

$$
E_{y}=-j \omega G_{y y}^{A} * J_{y}+\frac{1}{j \omega} \frac{\partial}{\partial y}\left[G_{q} * \nabla \cdot J\right]
$$

where $*$ implies convolution excepting where it is superscript denoting complex conjugation of a function.

To solve for the surface current density on the patch by the MoM, the first step is to expand the surface current densities in terms of a linear combination of the basis functions as follows:

$$
\begin{aligned}
& J_{x}=\sum_{n} A_{n} J_{x n}(x, y) \\
& J_{y}=\sum_{n} B_{n} J_{y n}(x, y)
\end{aligned}
$$

where $A_{n}$ and $B_{n}$ are the unknown coefficients of the basis functions, $J_{x n}$ and $J_{y n}$. Next we substitute (2) into (1) and test the resulting equations using some testing functions $T_{x m}$ and $T_{y m}$ and a suitable definition of inner product, e.g.,

$$
\langle f, g\rangle=\iint d x d y f^{*}(x, y) g(x, y) .
$$

Since the testing functions and the tangential electric fields have nonzero values over complementary regions, the lefthand sides of (1a) and (1b) become zero after the testing, and the following algebraic equations are obtained for the coefficients $A_{n}$ and $B_{n}$ :

$$
\begin{aligned}
\sum_{n} A_{n} & \left\{\left\langle T_{x m}, G_{x x}^{A} * J_{x n}\right\rangle\right. \\
& \left.+\frac{1}{\omega^{2}}\left\langle T_{x m}, \frac{\partial}{\partial x}\left[G_{q} * \frac{\partial}{\partial x} J_{x n}\right]\right\rangle\right\} \\
& +\sum_{n} B_{n}\left\{\frac{1}{\omega^{2}}\left\langle T_{x m}, \frac{\partial}{\partial x}\left[G_{q} * \frac{\partial}{\partial y} J_{y n}\right]\right\rangle\right\}=0 \\
\sum_{n} B_{n} & \left\{\left\langle T_{y m}, G_{y y}^{A} * J_{y n}\right\rangle\right. \\
+ & \left.\frac{1}{\omega^{2}}\left\langle T_{y m}, \frac{\partial}{\partial y}\left[G_{q} * \frac{\partial}{\partial y} J_{y n}\right]\right\rangle\right\} \\
+ & \sum_{n} A_{n}\left\{\frac{1}{\omega^{2}}\left\langle T_{y m}, \frac{\partial}{\partial y}\left[G_{q} * \frac{\partial}{\partial x} J_{x n}\right]\right\rangle\right\}=0 .
\end{aligned}
$$

The first inner product term is written below as an example

$$
\begin{aligned}
\left\langle T_{x m}, G_{x x}^{A} * J_{x n}\right\rangle & \\
= & \iint_{D(T)} d x d y T_{x m}(x, y) \iint_{D(B)} d x^{\prime} d y^{\prime} \\
& \cdot G_{x x}^{A}\left(x-x^{\prime}, y-y^{\prime}\right) J_{x n}\left(x^{\prime}, y^{\prime}\right)
\end{aligned}
$$

where $D(T)$ and $D(B)$ represent the domain of the testing and basis functions, respectively, and

$$
G_{x x}^{A}(\rho)=\frac{1}{4 \pi} \int_{-\infty}^{+\infty} d k_{\rho} k_{\rho} H_{o}^{(2)}\left(k_{\rho} \rho\right) \tilde{G}_{x x}^{A}\left(k_{\rho}\right) .
$$

In general, each inner product term in the spatial domain, e.g., the one given in (5), is a five-dimensional integral: one of these is associated with the Green's function itself which is an improper integral (Sommerfeld integral) over an infinite domain and is given in (6); two of these are convolution integrals; and, the remaining two are inner products. Since the numerical integration of the five-dimensional integrals is computationally intensive, the convolution integral over the Green's function and the basis functions is often transferred over to the basis and testing functions, enabling one to carry out this integral analytically. With this step, the order of integration can be reduced to three. In spite of this, the evaluation of the inner product is still very time-consuming because of the slow convergence behavior of the integrands of the Green's function.

\section{B. Spectral Domain Analysis}

The tangential electric field on the plane of the patch due to the patch currents $J_{x}$ and $J_{y}$ can be expressed in the spectral domain as

$$
\tilde{E}_{x}\left(k_{x}, k_{y}\right)=\tilde{Z}_{x x}\left(k_{x}, k_{y}\right) \tilde{J}_{x}\left(k_{x}, k_{y}\right)+\tilde{Z}_{x y}\left(k_{x}, k_{y}\right) \tilde{J}_{y}\left(k_{x}, k_{y}\right)
$$

$$
\tilde{E}_{y}\left(k_{x}, k_{y}\right)=\tilde{Z}_{y x}\left(k_{x}, k_{y}\right) \tilde{J}_{x}\left(k_{x}, k_{y}\right)+\tilde{Z}_{y y}\left(k_{x}, k_{y}\right) \tilde{J}_{y}\left(k_{x}, k_{y}\right)
$$

where implies Fourier transforms, and the electric field Green's functions $Z_{i j}$ in the spectral domain are expressible in closed-forms [8]. The application of the moment method starts with the expansion of the current densities as in (2), substitution of the Fourier transforms of these basis functions in (7), followed by the testing with the Fourier transforms of the testing functions. Following this procedure one arrives at the following algebraic equations:

$$
\begin{aligned}
& \sum_{n} A_{n}\left\langle\tilde{T}_{x m}, \tilde{Z}_{x x} \tilde{J}_{x n}\right\rangle+\sum_{n} B_{n}\left\langle\tilde{T}_{x m}, \tilde{Z}_{x y} \tilde{J}_{y n}\right\rangle=0 \\
& \sum_{n} A_{n}\left\langle\tilde{T}_{y m}, \tilde{Z}_{y x} \tilde{J}_{x n}\right\rangle+\sum_{n} B_{n}\left\langle\tilde{T}_{y m}, \tilde{Z}_{y y} \tilde{J}_{y n}\right\rangle=0
\end{aligned}
$$


where the inner products are defined over an infinite domain. The first inner product term is written as

$$
\begin{aligned}
\left\langle\tilde{T}_{x m}, \tilde{Z}_{x x} \tilde{J}_{x n}\right\rangle= & \iint_{-\infty}^{\infty} d k_{x} d k_{y} \tilde{T}_{x m}^{*}\left(k_{x}, k_{y}\right) \\
& \cdot Z_{x x}\left(k_{x}, k_{y}\right) \tilde{J}_{x n}\left(k_{x}, k_{y}\right) .
\end{aligned}
$$

Since the Green's functions in the spectral domain are expressible in closed forms, the elements of the MoM matrix become double integrals (9) over infinite ranges. However, one of the integrations could be transferred over to the finite domain by transforming the cartesian coordinates into polar coordinates. As a consequence, for microstrip geometries, the spectral domain moment method can be more efficient than the conventional spatial domain approach which requires the computation of triple integrals. The spectral domain approach is usually employed in conjunction with an acceleration technique in which the asymptotic part of the Green's function is subtracted from the original Green's function, and its contribution is calculated either analytically or in a numerically efficient manner [9], [10]. Even so, this computation is still expensive because the integrands are oscillatory functions of the spectral domain variables, and for the self-terms, for which the observation segment coincides with the source segment, the convergence is still quite slow.

\section{Closed-Form Green's Functions for the Vector and Scalar Potentials}

One remedy for the aforementioned convergence problem is to express the spatial domain Green's functions in closed forms so that the inner products become two-dimensional integrals over finite ranges (see Section II-A), and the time-consuming part of the moment method in the spatial domain, which entails the evaluation of the integral representations of the Green's functions, is completely avoided. The Sommerfeld integrals for the Green's functions corresponding to the vector and scalar potentials are written as

$$
G^{A, q}=\frac{1}{4 \pi} \int_{\mathrm{SIP}} d k_{\rho} k_{\rho} H_{o}^{(2)}\left(k_{\rho} \rho\right) \tilde{G}^{A, q}\left(k_{\rho}\right)
$$

1. Obtain the Green's functions for the vector and scalar potentials in the spectral domain.

2. Find the quasi-static images (real images) and their contributions by using the Sommerfeld identity.

3 . Find the surface-wave poles and calculate their contributions analytically.

4. Approximate the remaining integrand, which is now a smooth and relatively rapidly decaying function of the integration variable $k_{\rho}$ in (10), can be approximated in terms of complex exponentials using the least square Prony or the Pencil of Function method.

This technique is demonstrated, step by step, for a planar geometry with a substrate and a superstrate of arbitrary thicknesses and dielectric constants, as shown in Fig. 2.

\section{A. Green's Functions for the Vector and Scalar} Potentials in the Spectral Domain

For the geometry shown in Fig. 2, the Green's functions in the spectral domain can be written as

$$
\begin{aligned}
\tilde{G}_{A}^{x x} & =\frac{\mu}{j 2 k_{z i}} \tilde{T}_{\mathrm{TE}}(z) \\
\tilde{G}_{q} & =\frac{1}{\epsilon_{i} j 2 k_{z i}}\left[\tilde{T}_{\mathrm{TE}}+\frac{k_{z i}^{2}}{k_{\rho}^{2}}\left(\tilde{T}_{\mathrm{TE}}+\frac{1}{j k_{z i}} \frac{\partial}{\partial z} \tilde{T}_{\mathrm{TM}}\right)\right]
\end{aligned}
$$

where

$$
\begin{gathered}
\tilde{T}_{\mathrm{TE}}=\left[e^{-j k_{z i} z}+\bar{R}_{\mathrm{TE}}^{+} e^{-j k_{z i}\left(2 d_{i}-2 h-z\right)}+\bar{R}_{\mathrm{TE}}^{-} e^{-j k_{z i}(z+2 h)}\right] \\
\tilde{T}_{\mathrm{TE}}+\frac{k_{z i}^{2}}{k_{\rho}^{2}}\left(\tilde{T}_{\mathrm{TE}}+\frac{1}{j k_{z i}} \frac{\partial}{\partial z} \tilde{T}_{\mathrm{TM}}\right) \\
=\left[e^{-j k_{z i z}}+\left(\bar{R}_{\mathrm{TE}}^{+}+\bar{R}_{q}^{+}\right) e^{-j k_{z i}\left(2 d_{i}-2 h-z\right)}\right. \\
\left.+\left(\bar{R}_{\mathrm{TE}}^{-}+\bar{R}_{q}^{-}\right) e^{-j k_{z i}(z+2 h)}\right] \\
\bar{R}_{q}^{ \pm}=\frac{k_{z i}^{2}}{k_{\rho}^{2}}\left[\bar{R}_{\mathrm{TE}}^{ \pm}-\bar{R}_{\mathrm{TM}}^{ \pm}\right]
\end{gathered}
$$

$\bar{R}_{\mathrm{TM}, \mathrm{TE}}^{ \pm}(z)$

$$
\left.=\frac{ \pm \tilde{R}_{\mathrm{TM}, \mathrm{TE}}^{i, i \pm 1}+\tilde{R}_{\mathrm{TM}, \mathrm{TE}}^{i, i+1} \tilde{R}_{\mathrm{TM}, \mathrm{TE}}^{i, i-1} e^{-j k_{z} i 2(h+z)}}{1-\tilde{R}_{\mathrm{TM}, \mathrm{TE}}^{i, i+1} \tilde{R}_{\mathrm{TM}, \mathrm{TE}}^{i, i-1} e^{-j k_{z i} 2 d_{i}}}\right\} \begin{aligned}
& - \text { for TM } \\
& + \text { for TE }
\end{aligned}
$$

$$
\left.\bar{R}_{\mathrm{TM}, \mathrm{TE}}^{-}(z)=\frac{ \pm \tilde{R}_{\mathrm{TM}, \mathrm{TE}}^{i, i-1}+\tilde{R}_{\mathrm{TM}, \mathrm{TE}}^{i, i+1} \tilde{R}_{\mathrm{TM}, \mathrm{TE}}^{i, i-1} e^{-j k_{z i} 2\left(d_{i}-h-z\right)}}{1-\tilde{R}_{\mathrm{TM}, \mathrm{TE}}^{i, i+1} \tilde{R}_{\mathrm{TM}, \mathrm{TE}}^{i, 1} e^{-j k_{z} i 2 d_{i}}}\right\} \begin{aligned}
& - \text { for TM } \\
& + \text { for TE }
\end{aligned}
$$

where

$\tilde{G}^{A}$ is the Green's function of the vector potential in the spectral domain

$\tilde{G}^{q} \quad$ is the Green's function of the scalar potential in the spectral domain

$H_{o}^{(2)}$ is the Hankel function of the second kind

SIP stands for the Sommerfeld Integration Path.

The procedure for deriving closed-form expressions for the Green's functions entails the following steps: and $\tilde{R}_{\mathrm{TM}, \mathrm{TE}}^{i, i+1}$ are the generalized TM and TE reflection coefficients at the interface between regions $i$ and $i+1$, which are defined in terms of the Fresnel reflection coefficients [11]. The Green's functions given by (11a) and (11b) are obtained for the observation point $z \in\left[0, d_{i}-h\right]$.

\section{B. Quasi-Static Images and their Contributions}

To obtain the spatial domain counterparts of the spectral Green's functions given in (11a) and (11b), it is necessary to evaluate their inverse Hankel transforms, as for 


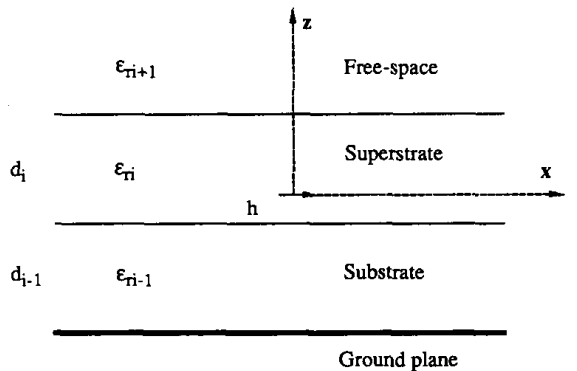

Fig. 2. Substrate-superstrate geometry.

instance in (10). In general, the inversion integral in (10) can not be evaluated analytically. However, for the quasistatic images that are exponential approximations of the spectral domain Green's functions as $k_{o} \rightarrow 0$, the inverse Hankel transform can be evaluated analytically by using the Sommerfeld identity. Since the quasi-static fields are defined in the range in which the observation distance is much smaller than the free-space wavelength $(\rho<<\lambda)$, they correspond to the asymptotic components $\left(k_{\rho} \rightarrow \infty\right)$ of the Green's functions in the spectral domain. Therefore, the subtraction of these quasi-static terms from the Green's functions (11a) and (11b) makes the remaining integrands of (10) decay faster for large $k_{\rho}$. From (12a) and (12b), we note that we only need the asymptotic terms of the reflection coefficients $R_{\mathrm{TE}}$ 's and $R_{q}$ 's, which are denoted here as $R_{\mathrm{TE} 0}$ and $R_{q 0}$, and are given by

$$
\left.\left.\begin{array}{rl}
\bar{R}_{\mathrm{TE} 0}^{+} \approx 0 & \approx \text { as } k_{o} \rightarrow 0 \\
\bar{R}_{\mathrm{TE} 0}^{-} & \approx-e^{-j k_{z i} d_{i}-1}
\end{array}\right\} \begin{array}{c}
\bar{R}_{q 0}^{+} \approx K_{i, i+1}\left(1-K_{i-1, i)} e^{-j 2 k_{z i}(h+z)}\right. \\
\bar{R}_{q 0}^{-} \approx-K_{i-1, i}\left[1-K_{i-1, i} e^{-j 2 k_{z i} d_{i-1}}\right. \\
\left.\quad+K_{i, i+1} e^{-j 2 k_{z i}\left(d_{i}-h-z\right)}\right]
\end{array}\right\} \text { as } k_{o} \rightarrow 0
$$

where $K_{i, i+1}=\left(\epsilon_{r i}-\epsilon_{r i+1}\right) /\left(\epsilon_{r i}+\epsilon_{r i+1}\right)$ and $K_{i-1, i}=$ $\left(\epsilon_{r i-1}-\epsilon_{r i}\right) /\left(\epsilon_{r i-1}+\epsilon_{r i}\right)$.

After extracting the contributions of the quasi-static components, the Green's functions, given in (10), can be rewritten as

$$
\begin{aligned}
G_{q 0}= & \frac{1}{4 \pi \epsilon_{i}} \frac{e^{-j k_{i} r_{d}}}{r_{d}}+\frac{K_{i, i+1}}{4 \pi \epsilon_{i}} \frac{e^{-j k_{i} r_{i 2}}}{r_{i 2}}-\frac{K_{i, i+1} K_{i-1, i}}{4 \pi \epsilon_{i}} \\
& \cdot \frac{e^{-j k_{i} r_{i 3}}}{r_{i 3}}-\frac{K_{i-1, i}}{4 \pi \epsilon_{i}} \frac{e^{-j k_{i} r_{i 4}}}{r_{i 4}}-\frac{\left(1-K_{i-1, i}^{2}\right)}{4 \pi \epsilon_{i}} \\
& \cdot \frac{e^{-j k_{i} r i 5}}{r_{i 5}}-\frac{K_{i, i+1} K_{i-1, i}}{4 \pi \epsilon_{i}} \frac{e^{-j k_{i} r_{i}}}{r_{i 6}} \\
r_{d}= & \sqrt{\rho^{2}+z^{2}} ; \quad r_{i 1}=\sqrt{\rho^{2}+\left(z+2 h+2 d_{i-1}\right)^{2}} ; \\
r_{i 2}= & \sqrt{\rho^{2}+\left(2 d_{i}-2 h-z\right)^{2}} ; \\
r_{i 3}= & \sqrt{\rho^{2}+\left(2 d_{i}+z\right)^{2}} ; \quad r_{i 4}=\sqrt{\rho^{2}+(z+2 h)^{2}} ; \\
r_{i 5}= & \sqrt{\rho^{2}+\left(2 d_{i-1}+z+2 h\right)^{2}} ; \\
r_{i 6}= & \sqrt{\rho^{2}+\left(2 d_{i}-z\right)^{2}} .
\end{aligned}
$$

Note that the first term in both (14a) and (14b) represents the direct field, i.e., the response of a point source in an infinite medium with wavenumber $k_{i}$, while the remaining terms in the expressions for the vector and scalar potentials are the real images, one for the former and five for the latter.

\section{Surface-Wave Poles and their Contributions}

It is well-known that the Sommerfeld integrals for layered media contain a certain number of poles and branch singularities and that these singularities are associated with the surface, leaky and lateral waves launched by the source. Among these, the surface waves play a rather significant role as they are guided along the interface without leaking energy. The corresponding pole singularities are located on the real axis of the $k_{\rho}$-plane and must be handled properly. Even if the integration path is deformed such that it is not too close to these pole singularities, their presence still affects the value of the integral for small values of $k_{\rho}$. It is helpful to extract these singularities from the integrand before employing Prony's method to approximate it, because this helps smooth the integrand

$$
\begin{aligned}
G_{A}^{x x}= & G_{A 0}^{x x}+\frac{\mu}{4 \pi} \int_{\mathrm{SIP}} d k_{\rho} k_{\rho} H_{0}^{(2)}\left(k_{\rho} \rho\right) \frac{\bar{R}_{\mathrm{TE}}^{+} e^{-j k_{z i}\left(2 d_{i}-2 h-z\right)}+\left(\bar{R}_{\mathrm{TE}}^{-}-\bar{R}_{\mathrm{TE} 0}^{-}\right) e^{-j k_{z i}(z+2 h)}}{j 2 k_{z i}} \\
G_{q}= & G_{q 0}+\frac{1}{4 \pi \epsilon_{i}} \int_{\mathrm{SIP}} d k_{\rho} k_{\rho} H_{0}^{(2)}\left(k_{\rho} \rho\right) \\
& \cdot \frac{\left(\bar{R}_{\mathrm{TE}}^{+}+\bar{R}_{q}^{+}-\bar{R}_{q 0}^{+}\right) e^{-j k_{z i}\left(2 d_{i}-2 h-z\right)}+\left(\bar{R}_{\mathrm{TE}}^{-}+\bar{R}_{q}^{-}-\bar{R}_{\mathrm{TE} 0}^{-}-\bar{R}_{q 0}^{-}\right) e^{-j k_{i i}(z+2 h)}}{j 2 k_{z i}}
\end{aligned}
$$

where

$$
G_{A 0}^{x x}=\frac{\mu}{4 \pi} \frac{e^{-j k_{i} r_{d}}}{r_{d}}-\frac{\mu}{4 \pi} \frac{e^{-j k_{i} r_{i 1}}}{r_{i 1}}
$$

and renders it easier to approximate via the Prony algorithm.

Since the surface-wave poles always occur in complex conjugate pairs, a typical pair can be represented mathe- 
matically as

$$
\frac{2 k_{\rho p}\left(\text { Residue at } k_{\rho \rho}\right)}{k_{\rho}^{2}-k_{\rho p}^{2}}
$$

where $k_{\rho p}$ is the surface-wave pole. By subtracting these poles from the integrands (13a) and (13b), and analytically evaluating their contribution via the residue calculus technique, we can derive the following representation for the spatial domain Green's function for the vector potential:

$$
G_{A}^{x x}=G_{A 0}^{x x}+G_{A s w}^{x x}+\frac{\mu}{4 \pi} \int_{-\infty}^{\infty} d k_{\rho} k_{\rho} H_{o}^{(2)}\left(k_{\rho} \rho\right) \frac{F_{1}\left(k_{\rho}\right)}{j 2 \mathrm{k}_{z i}}
$$

where

$$
\begin{aligned}
G_{A s W^{\prime}}^{x x}= & \frac{\mu}{4 \pi} \sum_{i=1}^{N \mathrm{TE}} \int_{-\infty}^{\infty} d k_{\rho} k_{\rho} H_{o}^{(2)}\left(k_{\rho} \rho\right) \frac{2 k_{\rho \rho(i)} \operatorname{Res} 1^{(i)}}{k_{\rho}^{2}-k_{\rho p(i)}^{2}} \\
= & \frac{\mu}{4 \pi}(-j 2 \pi) \sum_{i=1}^{N \mathrm{TE}} k_{\rho \rho(i)} H_{o}^{(2)}\left(k_{\rho p(i)} \rho\right) \operatorname{Res} 1^{(i)} \quad(16 \mathrm{a}) \\
F_{1}\left(k_{\rho}\right)= & \bar{R}_{\mathrm{TE}}^{+} e^{-j k_{z i}\left(2 d_{i}-2 h-z\right)}+\left(\bar{R}_{\mathrm{TE}}^{-}-\bar{R}_{\mathrm{TE} 0}^{-}\right) e^{-j k_{z i}(z+2 h)} \\
& -j 2 k_{z i} \sum_{i=1}^{N_{\mathrm{TE}}} \frac{2 k_{\rho p(i)} \operatorname{Res} 1^{(i)}}{k_{\rho}^{2}-k_{\rho p(i)}^{2}} \\
\operatorname{Res}^{(i)}= & \left.\frac{e^{-j k_{z i}\left(2 d_{i}-2 h-z\right)}}{j 2 k_{z i}}\right|_{k_{\rho}=k_{\rho p(i)}} \lim _{k_{\rho} \rightarrow k_{\rho \rho(i)}}\left(k \rho-k_{\rho p(i)}\right) \bar{R}_{\mathrm{TE}}^{+} \\
& +\left.\frac{e^{-j k_{z i}(2 h+z)}}{j 2 k_{z i}}\right|_{k_{\rho}=k_{\rho p(i)}} \lim _{k_{\rho} \rightarrow \rho p(i)}\left(k_{\rho}-k_{\rho p(i)}\right) \bar{R}_{\mathrm{TE}}^{-} .
\end{aligned}
$$

Similarly, for the Green's function for the scalar potential, we can write

$$
\begin{aligned}
G_{q} & =G_{q 0}+G_{q s w}+\frac{1}{4 \pi \epsilon_{\iota}} \int_{-\infty}^{\infty} d k_{\rho} k_{\rho} H_{o}^{(2)}\left(k_{\rho} \rho\right) \frac{F_{2}\left(k_{\rho}\right)}{j 2 k_{z i}} \\
G_{q s w} & =\frac{1}{4 \pi \epsilon_{\iota}} \sum_{i=1}^{N_{\mathrm{TE}}+N_{\mathrm{TM}}} \int_{-\infty}^{\infty} d k_{\rho} k_{\rho} H_{o}^{(2)}\left(k_{\rho} \rho\right) \frac{2 k_{\rho p(i)} \operatorname{Res} 2^{(i)}}{k_{\rho}^{2}-k_{\rho p(i)}^{2}} \\
& =\frac{1}{4 \pi \epsilon_{\iota}}(-j 2 \pi) \sum_{i=1}^{N \mathrm{TE}+N_{\mathrm{TM}}} k_{\rho p(i)} H_{o}^{(2)}\left(k_{\rho p(i)} \rho\right) \operatorname{Res} 2^{(i)}
\end{aligned}
$$

$$
\begin{aligned}
F_{2}\left(k_{\rho}\right)= & \left(\bar{R}_{\mathrm{TE}}^{+}+\bar{R}_{q}^{+}-\bar{R}_{q 0}^{+}\right) e^{-j k_{z i}\left(2 d_{i}-2 h-z\right)} \\
& +\left(\bar{R}_{\mathrm{TE}}^{-}+\bar{R}_{\bar{q}}-R_{q 0}-\bar{R}_{\mathrm{TE} 0}^{-}\right) e^{-j k_{z i}(z+2 h)} \\
& -j 2 k_{z i} \sum_{i=1}^{N_{\mathrm{TE}}+N \mathrm{NT}} \frac{2 k_{\rho p(i)} \operatorname{Res} 2^{(i)}}{k_{\rho}^{2}-k_{\rho p(i)}^{2}}
\end{aligned}
$$

$$
\begin{aligned}
& \operatorname{Res} 2^{(i)}=\left.\frac{e^{-j k_{z i}\left(2 d_{i}-2 h-z\right)}}{j 2 k_{z i}}\right|_{k_{\rho}=k_{\rho p(i)}} \\
& \left\{\begin{array}{c}
\lim _{k_{\rho} \rightarrow k_{\rho \rho \mathrm{TE}(i)}}\left(k_{\rho}-k_{\rho p \mathrm{TE}(i)}\right) \bar{R}_{\mathrm{TE}}^{+}\left(1+\frac{k_{z i}^{2}}{k_{\rho}^{2}}\right) \\
\lim _{k_{\rho} \rightarrow k_{\rho \rho \mathrm{TM}(i)}}\left(k_{\rho}-k_{\rho p \mathrm{TM}(i)}\right) \bar{R}_{\mathrm{TM}}^{+}\left(-\frac{k_{z i}^{2}}{k_{\rho}^{2}}\right)
\end{array}\right\} \\
& +\left.\frac{e^{-j k_{z i}(2 h+z)}}{j 2 k_{z i}}\right|_{k_{\rho}=k_{\rho p(i)}} \\
& \left\{\begin{array}{c}
\lim _{k_{\rho} \rightarrow k_{\rho \rho \mathrm{TE}(i)}}\left(k_{\rho}-k_{\rho p \mathrm{TE}(i)}\right) \bar{R}_{\mathrm{TE}}^{-}\left(1+\frac{k_{z i}^{2}}{k_{\rho}^{2}}\right) \\
\lim _{k_{\rho} \rightarrow k_{\rho \rho \mathrm{TM}(i)}}\left(k_{\rho}-k_{\rho p \mathrm{TM}(i)}\right) \bar{R}_{\mathrm{TM}}^{-}\left(-\frac{k_{z i}^{2}}{k_{\rho}^{2}}\right)
\end{array}\right\}
\end{aligned}
$$

The Green's functions given in (15) and (17) can be either evaluated numerically or approximated by their closedform expressions. The approximation procedure will be detailed in the next section.

\section{Approximation of the Remaining Integrands}

In this step, $F_{1}\left(k_{\rho}\right)$ and $F_{2}\left(k_{\rho}\right)$, given in (16b) and (18b), respectively, are approximated in terms of complex exponentials by using the least square Prony's method [12], [13]. In order to be able to use the Sommerfeld identity for the exponentials obtained from the approximations of $F_{1}\left(k_{\rho}\right)$ and $F_{2}\left(k_{\rho}\right)$, these exponentials should be functions of $k_{z i}$ which is a complex number in general. Since Prony's method is applicable to complex functions with real variables, we need to transform the complex variable $k_{z i}$ into a real variable $t$ by a parametric function [2], [3], defined as

$$
k_{z i}=k_{i}\left[-j t+\left(1-\frac{t}{T_{0}}\right)\right], \quad 0 \leq t \leq T_{0}
$$

which maps $t \in\left[0, T_{o}\right]$ into $k_{z i} \in\left[k_{i},-j k_{i} T_{o}\right]$. Since $T_{o}$ corresponds to $k_{i}\left[1+T_{o}^{2}\right]^{1 / 2}$ in the $k_{\rho}$-plane, the choice of $T_{o}$ is dependent upon the behavior of the integrands to be approximated for large $k_{\rho}$. Both $F_{1}\left(k_{\rho}\right)$ and $F_{2}\left(k_{\rho}\right)$ are uniformly sampled along the integration path $C$, which corresponds to the real variable $t$, and approximated in terms of exponentials of the variable $t$, or $k_{z i}$, as follows:

$$
F_{n}\left(k_{\rho}\right)=\sum_{i=1}^{N_{n}} a_{n i} e^{\alpha_{n i t}}=\sum_{i=1}^{N_{n}} b_{n i} e^{-\beta_{n i} k_{\overline{ }}}, \quad n=1,2
$$

where $b_{n i}$ and $\beta_{n i}$ are written in terms of $a_{n i}$ and $\alpha_{n i}$

$$
\beta_{n i}=\alpha_{n i} \frac{T_{o}}{k_{i}\left(1+j T_{o}\right)} ; \quad b_{n i}=a_{n i} e^{\beta_{n i} k_{i}}
$$

Next, the integrals in (15) and (17) are evaluated analyt- 
ically by using the Sommerfeld identity to yield

$$
\begin{aligned}
G_{A c i}^{x x} & =\frac{\mu}{4 \pi} \int_{C} d k_{\rho} k_{\rho} H_{o}^{(2)}\left(k_{\rho} \rho\right) \frac{F_{1}\left(k_{\rho}\right)}{j 2 k_{z i}}=\frac{\mu}{4 \pi} \sum_{i=1}^{N_{1}} b_{1 i} \frac{e^{-j k_{i} r_{1 i}}}{r_{1 i}} \\
G_{q c i} & =\frac{1}{4 \pi \epsilon_{\imath}} \int_{C} d k_{\rho} k_{\rho} H_{o}^{(2)}\left(k_{\rho} \rho\right) \frac{F_{2}\left(k_{\rho}\right)}{j 2 k_{z i}} \\
& =\frac{1}{4 \pi \epsilon_{i}} \sum_{i=1}^{N_{2}} b_{2 i} \frac{e^{-j k_{i} r_{2 i}}}{r_{2 i}}
\end{aligned}
$$

where $r_{1 i}$ and $r_{2 i}$ are complex numbers; hence, each of the terms in the above series is referred to as the contribution of a complex image.

In Prony's method, the required number of sampling points is at least twice the number of exponentials to be used in the approximation. If the number of sample points is chosen to be exactly twice the number of exponentials, the approximation will be exact only at the sampling points, and there will be no guarantee that it would be accurate elsewhere. It is therefore essential to take as many samples as necessary to ensure the capture of any rapid variation of the function being sampled. Consequently, the number of sampling points is usually taken to be much higher than twice the number of exponentials; this prompts us to use the least square Prony's method, which is designed to handle this situation.

\section{Results and Discussions}

Following the procedure described above, we have derived the closed-form Green's functions for the vector and scalar potentials for a horizontal electric dipole located at the interface between the substrate and superstrate as shown in Fig. 2. Their expressions are given by

$$
\begin{aligned}
G_{A}^{x x}= & \frac{\mu}{4 \pi} \frac{e^{-j k_{i} r_{d}}}{r_{d}}-\frac{\mu}{4 \pi} \frac{e^{-j k_{i} r_{i}}}{r_{i 1}}+\frac{\mu}{4 \pi}(-j 2 \pi) \sum_{i=1}^{N \mathrm{TE}} \\
& \cdot k_{\rho p(i)} H_{o}^{(2)}\left(k_{\rho p(i)} \rho\right) \operatorname{Res} 1^{(i)}+\frac{\mu}{4 \pi} \sum_{i=1}^{N_{1}} b_{1 i} \frac{e^{-j k_{i} r_{1 i}}}{r_{1 i}} \\
G_{q}= & \frac{1}{4 \pi \epsilon_{i}} \frac{e^{-j k_{i} r_{d}}}{r_{d}}+\frac{K_{i, i+1}}{4 \pi \epsilon_{i}} \frac{e^{-j k_{i} r_{i 2}}}{r_{i 2}}-\frac{K_{i, i+1} K_{i-1, i}}{4 \pi \epsilon_{i}} \frac{e^{-j k_{i} r_{i 3}}}{r_{i 3}} \\
& -\frac{K_{i-1, i}}{4 \pi \epsilon_{i}} \frac{e^{-j k_{i} r_{i 4}}}{r_{i 4}}-\frac{\left(1-K_{i-1, i}^{2}\right)}{4 \pi \epsilon_{i}} \frac{e^{-j k_{i} r_{i}}}{r_{i 5}} \\
& -\frac{K_{i, i+1} K_{i-1, i}}{4 \pi \epsilon_{i}} \frac{e^{-j k_{i} r_{i}}}{r_{i 6}}-\frac{j}{2 \epsilon_{\iota}} \sum_{i=1}^{N \mathrm{TE}+N_{\mathrm{TM}}} \\
& \cdot k_{\rho p(i)} H_{o}^{(2)}\left(k_{\rho p(i)} \rho\right) \operatorname{Res} 2^{(i)}+\frac{1}{4 \pi \epsilon_{i}} \sum_{i=1}^{N_{2}} b_{2 i} \frac{e^{-j k_{i} r_{2 i}}}{r_{2 i}}
\end{aligned}
$$

where $N_{\mathrm{TE}}$ and $N_{\mathrm{TM}}$ are the numbers of TE and TM surface-wave poles, and $N_{1}$ and $N_{2}$ are the numbers of com-

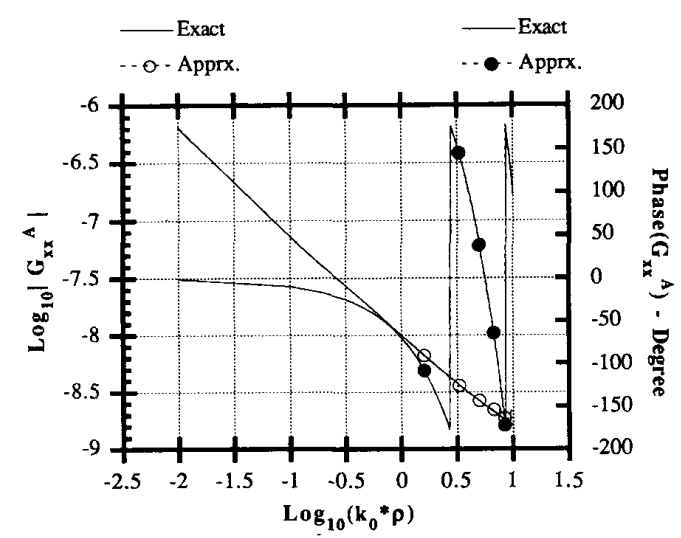

Fig. 3. The Green's function for the vector potential; magnitude and phase. $\epsilon_{r i}=12.5, d_{i-1}=0.07 \mathrm{~cm}, d_{i}=0.03 \mathrm{~cm}$, freq $=30 \mathrm{GHz}$.

plex images for the vector and scalar potentials, respectively.

For the sake of illustration, we have chosen GaAs $\left(\epsilon_{r}\right.$ $=12.5)$ with a thickness of $0.03 \mathrm{~cm}$ and teflon $\left(\epsilon_{r}=2.1\right)$ with a thickness of $0.07 \mathrm{~cm}$ as the superstrate and substrate materials, respectively. Two surface-wave poles have been found for this geometry at the frequency of 30 $\mathrm{GHz}$, one for TM at $k_{\rho}=7.38457$ and the other for TE at $k_{\rho}=6.49447$. Figs. 3 and 4 compare the magnitude and phase of the Green's functions obtained from the numerical integration of (15) and (17) with those computed from the closed-form expressions in (22a) and (22b) for an operating frequency of $30 \mathrm{GHz}$. The time savings realized from the use of the closed-form expression is substantial, approximately three orders of magnitude in this case, and yet the agreement is excellent between the two results.

In the example given above, the thickness of the substrate is about one-tenth of the wavelength in the dielectric medium of the substrate, which is not particularly thin. Thus, we consider another example of a microstrip geometry for which the dielectric constant and the substrate thickness are 4.0 and $0.02032 \mathrm{~cm}$, respectively. The frequency of operation is now chosen to be $1 \mathrm{GHz}$ and, for these parameters, the substrate thickness is only 0.00135 in terms of the wavelength in the medium and only one TM surface-wave pole exists, located at $k_{\rho}=0.20944$. In this example, we have used two and four complex images for the closed-form representations of the Green's functions for the vector and scalar potentials, respectively, i.e., have chosen $N_{1}=2$ and $N_{2}=4$ in (22a) and (22b). As can be seen from Figs. 5 and 6 , the closed-form Green's functions and the numerically integrated Sommerfeld integrals are found to be in very good agreement up to a moderate distance between the observation and source points, beyond which the closed-form approximation begins to deviate somewhat from the exact Green's functions. Although it is possible to employ asymptotic approximations [14] to derive more accurate representations of the Green's functions for large $\rho$, this is often unnecessary because the size of the conductors on a sub- 


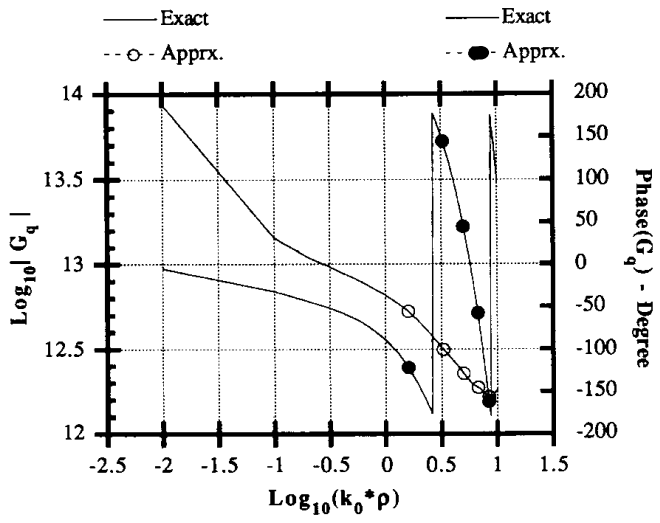

Fig. 4. The Green's function for the scalar potential; magnitude and phase. $\epsilon_{r i}=12.5, d_{i-1}=0.07 \mathrm{~cm}, d_{i}=0.03 \mathrm{~cm}$, freq $=30 \mathrm{GHz}$.

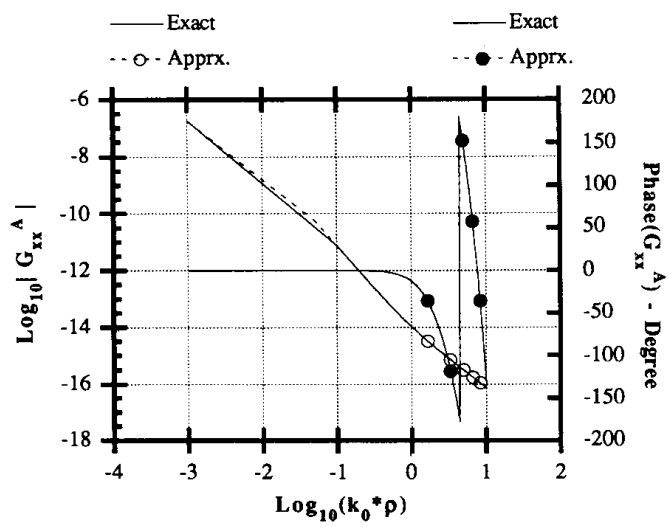

Fig. 5. The Green's function for the vector potential; magnitude and phase. $\epsilon_{r i-1}=4.0, \epsilon_{r i}=1.0, d_{i-1}=0.02032 \mathrm{~cm}$, freq $=1.0 \mathrm{GHz}$.

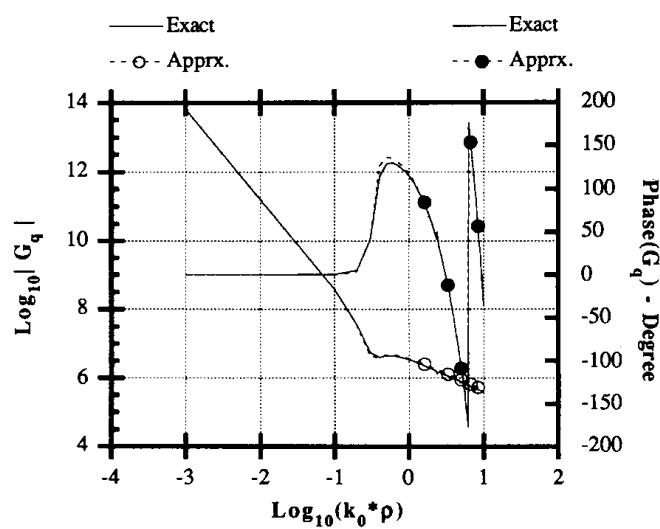

Fig. 6. The Green's function for the scalar potential; magnitude and phase. $\epsilon_{r i-1}=4.0, \epsilon_{r i}=1.0, d_{i-1}=0.02032 \mathrm{~cm}$, freq $=1.0 \mathrm{GHz}$.

strate is usually on the order of a wavelength or less, and the closed-form representations of the Green's functions are usually adequate.

We conclude this section by observing that the procedure given above is quite general and is applicable to a wide range of frequencies and material parameters, and yet its use results in savings of computation time in the calculation of the Green's functions as well as in the application of the MoM to microstrip problems.

\section{CONCLUSIONS}

In this paper we have presented a numerically-efficient technique for deriving closed-form approximations for the spatial domain Green's functions for microstrip geometries that find important applications in the numerical modeling of microstrip antennas and circuits. The accuracy of the procedure has been demonstrated via representative numerical examples.

\section{ACKNOWLEDGMENT}

The work reported in this paper was supported in part by the Joint Services Electronics Program under Grant N00014-90-J-1270. Support from the National Center for Supercomputing Applications at the University of Illinois for computer time on the Cray/YMP supercomputer is also acknowledged.

\section{REFERENCES}

[1] A. Sommerfeld, Partial Differential Equations in Physics. New York: Academic Press, 1949

[2] Y. L. Chow, J. J. Yang, D. H. Fang, and G. E. Howard,' Closedform spatial Green's function for the thick substrate," IEEE Trans. Microwave Theory Tech., vol. 39, pp. 588-592, Mar. 1991

[3] D. G. Fang, J. J. Yang and G. Y. Delisle, "Discrete image theory for horizontal electric dipoles in a multilayered medium," Proc. Inst. Elec. Eng., vol. 135, Pt. H, Oct. 1988.

[4] R. F. Harrington, Field Computation by Moment Methods. New York: MacMillan; Florida; Krieger Publishing, 1983.

[5] T. Itoh and R. Mittra, "Spectral domain approach for calculating the dispersion characteristics of microstrip line," IEEE Trans. Microwave Theory Tech., vol. MTT-21, pp. 496-499, 1973.

[6] D. C. Chang, D. I. Wu and J. X. Zheng, "Numerical modeling of passive networks and components in monolithic microwave integrated circuits (MMICs)," Directions in Electromagnetic Wave Modeling. H. L. Bertoni and L. B. Felsen, Eds., New York: Plenum, 1991.

[7] S. Barkeshli, P. H. Pathak, and M. Marin, "An asymptotic closedform microstrip surface Green's function for the efficient moment method analysis of mutual coupling in microstrip antennas," IEEE Trans. Antennas Propagat., vol. 38, pp. 1374-1383, Sept. 1990

[8] T. Itoh, "Spectral domain immittance approach for dispersion characteristics of generalized printed transmission lines," IEEE Trans Microwave Theory Tech, vol. MTT-28, pp. 733-736, July 1980.

[9] S. M. Wright, "Efficiency and analysis of infinite microstrip arrays on electrically thick substrate," Ph.D. dissertation, University of Illinois at Urbana-Champaign, 1984.

[10] M. I. Aksun, S. L. Chuang and Y. T. Lo, "Analysis of a slot excited by a semi-infinite microstrip transmission line," J. Electromagn. Waves Appl., to be published.

[11] W. C. Chew, Waves and Fields in Inhomogeneous Media. New York: Van Nostrand Reinhold, 1990.

[12] S. L. Marple, Digital Spectral Analysis with Applications. Englewood Cliffs, NJ: Prentice-Hall, 1987.

[13] F. D. Hildebrand, Introduction to Numerical Analysis. New York: McGraw-Hill, 1956.

[14] S. Barkeshli and P. H. Pathak, "Closed-form asymptotic representations for the grounded planar single and double layer material slab Green's functions and their applications in the efficient analysis of arbitrary microstrip geometries,"' Directions in Electromagnetic Wave Modeling, H. L. Bertoni, and L. B. Felsen, Eds., New York: Plenum, 1991 


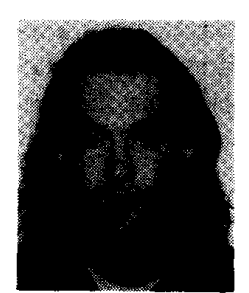

M. Irsadi Aksun recejved B.Sc. and M.Sc. degrees in electrical engineering from the Middle East Technical University, Ankara, Turkey, in 1981 and 1983, respectively, and the Ph.D. de gree in electrical and computer engineering from the University of Illinois at Urbana-Champaign in 1990. Currently, he is a Post doctoral fellow at the University of Illinois, Urbana-Champaign.

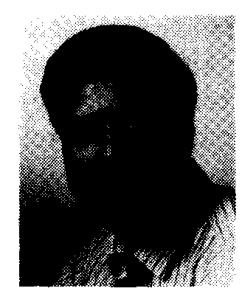

Raj Mittra (S'54-M'57-SM'69-F'71) is the Director of the Electromagnetic Communication Laboratory of the Electrical and Computer Engineering Department and Research Professor of the Coordinated Science Laboratory at the University of Illinois. He is a Past-President of AP-S, and he has served as the editor of the Transactions of the Antennas and Propagation Society. He won the Guggenheim Fellowship Award in 1965 and the IEEE Centennial Medal in 1984. He has been a Visiting Professor at Oxford University, Oxford,
England and at the Technical University of Denmark, Lyngby, Denmark. Currently, he serves as the North American editor of the journal AEÜ. He is President of RM Associates, which is a consulting organization providing services to several industrial and governmental organizations.

His professional interests include the areas of computational electromagnetics, electromagnetic modeling of electronic packaging, radar scattering, satellite antennas, microwave and millimeter wave integrated circuits, frequency selective surfaces, EMP and EMC analysis, and remote sensing. 